Published in final edited form as:

Epilepsy Res. 2012 August ; 101(1-2): 122-128. doi:10.1016/j.eplepsyres.2012.03.011.

\title{
Recruitment for Genetic Studies of Epilepsy
}

\author{
Sylwia Misiewicz ${ }^{1}$ and Melodie R. Winawer ${ }^{2}$ \\ ${ }^{1}$ GH Sergievsky Center, Columbia University, New York, NY \\ ${ }^{2}$ Department of Neurology, Columbia University, New York, NY
}

\section{Abstract}

Virtually nothing has been published about recruitment of adults with sporadic temporal lobe epilepsy (TLE) for genetic studies. We examined eligibility, recruitment, participation rates, and reasons for exclusion in a genetic study of TLE. Participants with non-acquired TLE with onset $\leq$ 35 were recruited through review of records and screening of incoming patients at Columbia University Medical Center (CUMC). Eligible patients were asked to participate in an interview about seizures and give a blood sample for DNA extraction. Medical records were sought for each participant. Of 2,974 patients screened 252 (8.5\%) were eligible, and 40 (15.9\% of eligible) participated. Leading reasons for ineligibility included an antecedent cause of epilepsy, syndrome other than TLE, and seizure onset after age 35. Those declining participation cited concerns about confidentiality, lack of compensation, and fear of phlebotomy. Although TLE is common and patients were recruited from a major surgical epilepsy center, a small proportion of potential participants participated. Large numbers need to be screened to reach the target sample size. Obtaining permission from treating physicians to contact their patients directly can improve recruitment. Saliva DNA collection, monetary incentives and patient education can improve participation. This information can facilitate study design in epilepsy genetics.

\section{Keywords}

Temporal lobe; Epilepsy; Recruitment; Participation; Genetics; Epidemiology

\section{INTRODUCTION}

Temporal lobe epilepsy (TLE) is the most common partial epilepsy in adults (Engel, 2001). Causative lesions or environmental antecedents are often identified including tumors, head trauma, and neuronal migration disorders, but there is substantial evidence for a genetic contribution to TLE (Ottman, 1989, Baulac et al., 2001, Ottman, 2001, Vadlamudi et al., 2003, Hedera et al., 2007). Consequently, TLE is frequently targeted for genetic studies, but barely any literature exists that examines recruitment and participation rates of affected individuals. This information can help develop effective recruitment strategies and

\footnotetext{
(C) 2012 Elsevier B.V. All rights reserved.

*Contact information: Melodie Winawer, 630 W. $168^{\text {th }}$ Street, P\&S Box 16, New York, NY 10032, Tel. 212-342-1336, mw211@columbia.edu.

Disclosure

The authors have no conflicts of interest to disclose. We confirm that we have read the Journal's position on issues involved in ethical publication and affirm that this report is consistent with those guidelines.

Publisher's Disclaimer: This is a PDF file of an unedited manuscript that has been accepted for publication. As a service to our customers we are providing this early version of the manuscript. The manuscript will undergo copyediting, typesetting, and review of the resulting proof before it is published in its final citable form. Please note that during the production process errors may be discovered which could affect the content, and all legal disclaimers that apply to the journal pertain.
} 
maximize participation (Ottman et al., 2005). Projection of participation rates and determination of the overall number of people to screen in order to achieve a target sample size are essential for design of similar studies and preparation of grant proposals.

After performing comprehensive literature search, we were unable to find any studies examining recruitment rates in genetic studies of sporadic epilepsy. The few studies that examined recruitment rates in familial epilepsy report varying findings. Ottman and colleagues (Ottman et al., 2005) examined recruitment of families for a genetic study of epilepsy and found $54 \%$ of screened families to be eligible, and $54 \%$ of the eligible families were successfully enrolled. Recruitment for family studies creates special difficulties because: (a) more than one person in the family has to be affected to be eligible, (b) other family members have to agree to participate, and (c) the proband is often left with the task of recruiting relatives. However, members of families with multiple affected individuals may be more motivated to participate in genetic studies because of the shared disease and perceived genetic contribution. Some individuals may be pressured or persuaded by their relatives to participate (Haddow, 2009). Recruitment rates for a genetic family study of epilepsy in a pediatric population have been reported in which both children and parents were enrolled(Dlugos et al., 2005). One hundred and sixty-eight children met the inclusion criteria; $137(82 \%)$ participated and 31(18\%) refused. Fear of phlebotomy was cited as the main reason for refusal. Here we describe recruitment strategies and enrollment results in a genetic study of sporadic TLE in adult subjects, examine reasons for exclusion, and discuss issues that may arise during recruitment.

\section{METHODS}

The data presented here was collected from August 2009 to December 2010 as part of The Partial Epilepsy Study of Columbia University, a genetic association (case-control) study of epilepsy. For this period, the recruitment was limited to patients with clearly defined TLE. In an attempt to speed recruitment, after December 2010 inclusion criteria were broadened to include all non-acquired focal epilepsy. Here we focus attention specifically on recruitment of TLE patients in the first phase of the study. TLE is a syndrome of great interest in the epilepsy community overall and in the area of epilepsy genetics in general because it is common, often difficult to treat, and has been shown to have a genetic contribution (Vadlamudi et al, 2003; Hwang and Hirose, 2011). The study protocol was approved by the Columbia University Medical Center (CUMC) Institutional Review Board (IRB).

\section{ELIGIBILITY CRITERIA}

To be eligible for the study, individuals had to have non-acquired TLE. We included those with mesial temporal sclerosis (MTS) and focal cortical dysplasia (FCD). Other criteria included a good quality MRI and EEG, no known disease-associated genetic mutations, and age of onset $\leq 35$. Previous research suggests that individuals with onset of seizures over the age of 35 are more likely have epilepsy with symptomatic etiology(Hauser et al., 1993, Ottman et al., 1996).

If available, affected siblings of probands were recruited if they had non-acquired epilepsy, though not necessarily TLE. As for the proband, the age of seizure onset for siblings had to be on or before age 35 . We excluded identical twins.

Attempts were made to recruit controls for each proband. Controls were drawn from married-in unaffected individuals from the proband's family, or if none were available, a friend of the proband with no history of seizures was recruited as a control. 


\section{ASCERTAINMENT SOURCES}

Potentially eligible participants were ascertained from the Columbia University Medical Center (CUMC) Comprehensive Epilepsy Center (CEC)including the Epilepsy Monitoring Unit (EMU), and the CUMC adult epilepsy clinic. Another source of recruitment was the CUMC Neurological Institute Department of General Neurology (NI2) which provides ambulatory care and management of acute and chronic neurological conditions. A portion of NI2 patients have epilepsy diagnoses. We also solicited self-referrals through flyers and brochures distributed around CUMC, primarily in the Neurological Institute. Through the proband we ascertained affected siblings and controls when they were available.

The referral patterns and catchment areas for the CEC and NI2 are similar to each other; the CPMC adult epilepsy clinic represents a different demographic. The majority of patients come to the CEC and NI2 from the wider metropolitan New York area--New York City, northern New Jersey, Connecticut and Westchester County. The CEC is made up of physicians in private practice and is primarily a referral-based tertiary care practice. The CEC patient population is mostly privately insured and self- paying $(32.7 \%$ and $39.0 \%$ respectively). In the CEC a relatively small number of patients (19.2\%) have Medicare and 7.6\% Medicaid as their primary insurance. In NI2 an even larger proportion of patients is privately insured (41.5\%), 21.7\% pay out of pocket and 33.2\% have Medicare. The racial/ ethnic make-up of the CEC/NI2 population is primarily White non-Hispanic with a smaller number of Hispanic patients.

The adult epilepsy clinic is staffed by the epilepsy fellows and neurology residents under the supervision of epilepsy attending physicians. The majority (80\%) of patients in the clinic come from the local neighborhoods of Washington Heights and Harlem, which have a large concentration of Hispanic and black non-Hispanic individuals; the other 20\% come from other boroughs of New York, primarily Brooklyn and the Bronx. The participants from the adult epilepsy clinic therefore more accurately reflect the local racial/ethnic demographic of Washington Heights and Harlem than the CEC and NI2. Most patients in the adult epilepsy clinic have Medicaid as their primary source of payment (90-95\%) with the remaining ( $5 \%)$ receiving Medicare.

The EMU population is referred from the CEC, NI2, and the adult epilepsy clinic, and the demographic distribution is therefore a combination of the other three sources. At the EMU, $51.9 \%$ of patients have private insurance, $25.4 \%$ are covered under Medicare and $18.6 \%$ have Medicaid.

We enrolled only those participants who were willing to have a blood draw completed either at CUMC or one of the Shiel laboratory (Shiel Medical Laboratory, Brooklyn, NY) locations in the New York Tri-state area.

\section{IDENTIFICATION AND CONTACT OF POTENTIAL PARTICIPANTS}

Potential participants were identified through chart review of incoming patients and consultation with treating physicians. To identify eligible patients at the CEC, EMU, and adult epilepsy clinic, we systematically reviewed charts of patients who were scheduled to be seen by a neurologist or admitted to the EMU on a given day. In the CEC and adult epilepsy clinic, the potentially eligible patient was approached by the physician who asked if the affected individual would be interested in contributing to our study. In the EMU, either the physician or one of the hospital staff providing care to the patient was asked to approach the patient regarding participation.

In NI2, we reviewed files of patients with epilepsy diagnoses seen up to two years prior to review. We obtained a list of all patients who were given ICD-9 codes consistent with an 
epilepsy diagnosis and only those patients were screened for eligibility. This allowed us to quickly eliminate patients seen in NI2 for neurological conditions other than epilepsy. The treating physician was then asked to initiate contact or approach the potentially eligible patient about our study during the scheduled office visit. Once the patient agreed to discuss the study, the research coordinator administered the eligibility screen and obtained consent according to CUMC IRB and HIPAA regulations. Some patients recruited through the CEC, adult epilepsy clinic, or NI2 chose to be interviewed and have their blood drawn right away. Others chose to come back to CUMC for a blood draw and an interview at a more convenient time. We offered participants the option of completing the interview over the phone and having a blood sample taken either at CUMC or by one of the Shiel laboratories located in the New York Tri-state area. For those participants who were currently at the EMU, both the blood draw and interview were completed during their stay at the hospital.

A different procedure was initiated for one of the treating CUMC neurologists from NI2, at his request. After screening his patients for eligibility, we provided him with a list of those who met our eligibility criteria. Once any of those patients came for a visit, the treating physician described the study and gave our contact information to interested patients. It was then left up to the patient to contact the research coordinator if he or she wished to participate. In those cases, after participant initiated contact with the research coordinator, the same procedure was followed in regards to consent and scheduling an interview and blood draw as for all others.

If we found that the proband had a sibling with epilepsy, we asked the proband for permission to contact the affected sibling to describe the study and assess the sibling's interest in participation. If permission was given, we then contacted the sibling, obtained consent and screened for eligibility. The same options were given to the sibling as to the proband regarding scheduling a blood draw and an interview. We also asked the proband for permission to contact any unrelated unaffected individuals, including married-in individuals, as controls.

\section{DATA COLLECTION}

Eligible patients who agreed to participate were asked to complete a series of structured and semi-structured questionnaires that took approximately one and a half hours to administer, and to undergo a blood draw for collecting a DNA sample. The following questionnaires were administered in this order: an eligibility screening instrument, sociodemographic form, family composition form including family pedigree, and a diagnostic interview. The main purpose of the eligibility screening instrument was to gather information regarding the participant's history of seizures or epilepsy, and help rule out any antecedent cause of epilepsy. The sociodemographic form included questions about age, gender, and education, as well as the race and ethnicity of the proband and grandparents on each side of the family. The sociodemographic form was also used to collect contact information for possible controls and informants who could answer questions about participant's seizures. Information about the possibility of seizures in other family members, and about unaffected $1^{\text {st }}$ degree relatives was gathered using a family composition form and family pedigree. The diagnostic interview consisted of both open-ended and multiple response choice questions regarding seizure types, frequency, specific seizure symptoms, triggers, a screen for status epilepticus, and also included information about alcohol intake and migraines. To facilitate classification according to ILAE syndromes(ILAE Commission, 1989)questions also addressed nocturnal or diurnal seizure occurrence, the occurrence of seizures on awakening, and seizure frequency. This diagnostic interview has previously been found to be a reliable instrument for gathering accurate information for the diagnosis and classification of epilepsy (Ottman et al., 1990, Ottman et al., 1993). 
All affected participants were asked for permission to access their medical records including MRI/EEG, Epilepsy Monitoring Unit (EMU) admissions, doctors' office notes, operative reports, and any other medical records relevant to the epilepsy diagnosis. Patients who agreed to have their medical records reviewed had to sign a medical release form. The appropriate HIPAA form was also signed by all participants. Refusal to access medical records did not affect eligibility.

If the participant signed the release of medical records form, medical records were obtained from the treating physician. The medical record abstraction form was used to record participant's clinical information from medical records including etiology, risk factors, seizure types, syndromes, semiology, neurological exam findings, and history of status epilepticus. For participants who had epilepsy surgery, a surgical form was completed that included information about neuropsychological evaluation, WADA testing, surgical procedure and electrode types and placement, seizure frequency before and after surgery, post-operative complications, postsurgical follow-up, pathology, and post-operative MRI.

Controls, after signing the appropriate HIPAA and consent forms, were asked to complete a 15-minute interview that included a separate eligibility screen and the sociodemographic form. They were also asked to undergo a blood draw for a DNA sample.

\section{RESULTS}

A total of 2,974 patients were screened over 15 months; 1906 (64.1\%) from the CEC including the adult epilepsy clinic and EMU, 161(5.4\%) from NI2, and 907(30.5\%) from the additional neurologist who requested a different initial contact protocol.

From the CEC, EMU and adult epilepsy clinic, only 116/1906 (6.1\%) were eligible; from NI2, 6/161 (3.7\%) were eligible, and from the additional NI2 neurologist 130/907 (14.3\%) were eligible to participate. From all sources, 252 out of $2974(8.5 \%)$ were found to be eligible. Of eligible patients, only 40/252 (15.9\%) participated.

36 out of 40 participants (90.0\%) signed the release of medical records form that allowed us to request medical records from the treating neurologist.

For the 2,722 patients who were screened but found to be ineligible to participate because they did not meet the inclusion criteria, the reasons for exclusion included: acquired/ symptomatic epilepsy $(1,046 ; 38.4 \%)$, localization/syndrome other than TLE $(792 ; 29.1 \%)$, age of onset over $35(376 ; 13.8 \%)$, seen by physician for reason other than seizure (250; $9.2 \%)$, no clear diagnosis $(150 ; 5.5 \%)$, single unprovoked seizure $(54 ; 2.0 \%)$, non-epileptic event $(46 ; 1.7 \%)$, and acute symptomatic seizures only $(8 ; 0.3 \%)$ (Figure 1$)$. The 250 individuals who never experienced a seizure were seen in the CUMC for another reason such as syncope, sleep disorder or a movement disorder. Of the 2,722 ineligible, 582(21.4\%) patients met more than one exclusion criterion.

Of the 792 patients who did not meet eligibility criteria due to epilepsy diagnosis other than TLE, the diagnoses were as follows: $382(48.2 \%)$ had generalized epilepsy (excluding JME), $90(11.4 \%)$ had juvenile myoclonic epilepsy (JME), 95 (12.0\%) had frontal lobe epilepsy, 68 (8.6\%) had symptomatic generalized epilepsy including Lennox-Gastaut syndrome, 42 (5.3\%) had multifocal epilepsy, 20(2.5\%) had occipital lobe epilepsy, $9(1.1 \%)$ had parietal lobe epilepsy, and $86(10.9 \%)$ had unclear diagnosis (whether localization-related epilepsy or generalized epilepsy).

From the total of 38 recruited probands, two (5.3\%) reported having affected siblings with non-acquired epilepsy. One proband reported having a sibling with symptomatic epilepsy 
due to brain tumor. We successfully recruited one family with 3 affected siblings, all of whom participated. Another 11 participants (28.9\%) reported having an affected family member other than a sibling, including distant relatives.

$62.5 \%$ of participants were women (25/40) and $37.5 \%$ were men (15/40). Overall, more Caucasian subjects (60\%) participated than those from other racial/ethnic subgroups (40\%) (see Table 1).

More than half of eligible patients did not participate because they had not yet been contacted by their physician ( $\mathrm{N}=195 ; 77.4 \%)$. Other reasons included refusal (12), and living out of the catchment area for the blood draw (5) (Figure 2). We are still attempting to enroll potentially eligible participants who have not yet been contacted by their physician regarding participation in the study.

\section{DISCUSSION}

In our study overall, $8.5 \%$ of screened patients were eligible to participate and the most common reason for exclusion of patients was known or presumed symptomatic etiology. Of the eligible patients, $15.9 \%$ participated. The two major problems that impaired recruitment were the small number of eligible participants and difficulty initiating contact with those who appeared to be eligible.

Waiting for physicians to contact their patients prior to contact by research staff created the greatest obstacle to recruitment. Obtaining permission from treating physicians to contact patients directly can streamline enrollment, but is not acceptable for all IRBs, physicians, and patients. In general the researcher is prohibited from directly contacting potential research subjects. Most IRBs require that any contact with patients must be done by the treating physician although some would permit that both the researcher and treating physician contact the patient jointly (e.g., through a letter) or allow the researcher to reference the physician during contact with patient when appropriate HIPAA authorization is provided.

Careful phenotype definition is important in genetic studies for creating a group of wellcharacterized subjects more likely to be homogenous, but narrow definitions of categories may slow recruitment. Broadening recruitment to additional populations may be helpful if the aims of the study support a broader phenotype. For the purpose of increasing our recruitment we expanded the inclusion criteria to all localization-related epilepsy (LRE), maintaining all other criteria. This decision was based on existing evidence suggesting that some genetic influences are shared by all LRE (Ottman, 1998; Ottman, 2001; Winawer et al., 2003; Mefford et al., 2010). Relaxing our eligibility criteria allowed us to accelerate recruitment.

We recognize that because our recruitment was limited to the CUMC patient population, the findings may not be applicable to the general population. However, our results allow us to make predictions about similar populations. Tertiary care epilepsy centers are often the site of recruitment for well-characterized patients in genetic studies, particularly those with temporal lobe epilepsy, as they will have been referred to surgical or tertiary care centers. This is the case, for example, in the Epilepsy Phenome/Genome project, the largest epilepsy genetics consortium in the United States, which recruits from 27 epilepsy centers nationally and internationally (www.EPGP.org). There are several other significant advantages to our ascertainment strategy. The combination of the very different sources for ascertainment-CEC/NI2 vs. the adult epilepsy clinic-- allowed us to sample a broad socioeconomic and racial/ethnic range of participants. In addition, recruitment through a tertiary care epilepsy center has its advantages because of the availability of more accurate and complete clinical 
information including detailed clinical medical records, EEG, EMU reports, MRI and surgical/pathologic reports. Access to medical records is essential when specific eligibility criteria have to be met for enrollment, and when accurate and detailed phenotyping is sought. Recruiting from CUMC allowed a high level of phenotypic accuracy that is of critical importance in the identification of genes underlying the complex epilepsies (Winawer, 2006).

Community-based studies are valuable because they allow greater generalizability, but they are also less likely to allow for the degree of detailed phenotyping permitted by genetic studies based at tertiary care referral centers. There are substantial potential advantages to community-based genetic studies, but these must be balanced by the quality of information that is available for assessing eligibility and delineating a clear and detailed phenotype.

We could not systematically record reasons for refusals because we could not contact anyone who initially did not give permission to be approached regarding the study. Physicians at the CEC listed the most common reasons for refusal when approaching patients regarding participation: lack of interest from individuals whose epilepsy is under control, fear of phlebotomy, lack of compensation, and concerns about privacy and confidentiality. Similar reasons for refusal were expressed by several patients who, after initially agreeing to speak to us regarding the study, declined to participate after learning more about it. One person wanted to be compensated for time devoted to the study, including transportation and wage reimbursement. Another patient refused due to privacy and confidentiality issues, unwillingness to have a blood sample taken, and lack of compensation. Several others expressed lack of interest in participating in general, especially without any direct benefit to their own health or care management.

A total of 12 potentially eligible subjects refused to participate. Excluding the patients of the one physician with a different approach to recruitment, only six patients were eligible from NI2. Only one of the eligible patients was approached by the treating physician to request participation and that subject participated. There were no refusals from NI2. All but one patient referred from the physician with the different recruitment approach participated; this patient later refused because of the expectation for financial compensation that was not in keeping with our protocol. All other refusals came from the CEC $(\mathrm{N}=7)$, EMU $(\mathrm{N}=1)$, and adult epilepsy clinic $(\mathrm{N}=3)$.

To address some of those concerns, such as fear of phlebotomy, researchers can consider less invasive alternatives for DNA extraction such as use of saliva samples. Although blood is the preferred method for obtaining DNA for both its quality and quantity, a saliva sample has been found to provide more than adequate DNA for genetic analysis (Rylander-Rudqvist et al., 2006). It is less invasive than a blood draw and therefore preferred by participants. Additionally, some people might be more likely to participate if a monetary incentive is offered (Bhatti et al., 2009, Doody et al., 2003, Bentley and Thacker, 2004, Kaufman et al., 2008).

Recruitment of controls proved to be difficult as many participants did not provide us with a referral for married-in controls or non-blood related individuals. The most common reason was lack of interest from those not directly affected by epilepsy especially given the study requirement for a blood draw. Instead of relying on unrelated controls, using existing biospecimens from control research repositories may be more efficient for such studies.

Over the past two decades, declining participation rates in research studies have been reported (Galea and Tracy, 2007). One reason cited is the increasing number of requests to participate in research studies and the greater demands on potential participants, such as lengthy survey assessments, biologic sampling and ongoing follow-up (Galea and Tracy, 
2007). Contacting individuals has become more challenging than ever due to what has been called an "oversurveyed" society (Galea and Tracy, 2007, Groves et al., 1992, Schleifer, 1986). Aggressive telemarketing has increased the number of people who screen their calls and more people are likely to have an unlisted phone number (Galea and Tracy, 2007, Remington, 1992). Changes in lifestyle have also contributed to difficulty in finding and contacting potential participants. Although the number of hours worked per week has remained fairly steady in the U.S. in the past few years, the proportion of people who work more than 50 hours per week has grown (Galea and Tracy, 2007). In the past few decades, women have been more likely to join the workforce, which diminishes the chances of reaching anyone at home (Galea and Tracy, 2007). Those issues should be taken into consideration when selecting methods of ascertainment to minimize cost and increase efficiency of recruitment. Selecting appropriate methods of contact and referral are also important (Ottman et al., 2005).

In conclusion, recruitment for genetic studies of partial epilepsy can be time consuming and laborious. Although TLE is the most common type of partial epilepsy, even at a major academic surgical epilepsy center a small fraction of patients screened are eligible and participate. Previous publications describing recruitment and participation in genetic studies of various disorders indicate several emerging themes. Female sex and older age have been found to be associated with higher participation rate (Hara et al., 2010). Also, altruism has been shown to be a key motivation to participation (Kirkland et al., 2009). Some of the most common concerns cited across studies including the study described here were participant's perceived lack of knowledge about genetics, concerns about misuse of genetic materials, commercialization of participant data, and privacy(Galea and Tracy, 2007, Bentley and Thacker, 2004). Community-based approaches have been found to enhance recruitment of minorities who are underrepresented in genomics-related research, including African Americans(Johnson et al., 2009, Murphy and Thompson, 2009, Murphy et al., 2009). Investigators can begin to work towards increasing recruitment and participation rates by educating the lay audience about genetic research, addressing concerns about privacy and confidentiality, and building trust. With these efforts, the scientific community can begin to dispel negative views towards genetic research and increase public interest and willingness to participate in genetic studies.

\section{Acknowledgments}

This work was supported by NIH grant K02 NS050429.

IRVING INSTITUTE ACKNOWLEDGMENT:

The project described was supported by the National Center for Research Resources and the National Center for Advancing Translational Sciences, National Institutes of Health, through Grant Number UL1 RR024156. The content is solely the responsibility of the authors and does not necessarily represent the official views of the NIH.

\section{References}

Baulac S, Picard F, Herman A, Feingold J, Genin E, Hirsch E, Prud'homme JF, Baulac M, Brice A, Leguern E. Evidence for digenic inheritance in a family with both febrile convulsions and temporal lobe epilepsy implicating chromosomes 18qter and 1q25-q31. Ann Neurol. 2001; 49:786-92. [PubMed: 11409431]

Bentley JP, Thacker PG. The influence of risk and monetary payment on the research participation decision making process. J Med Ethics. 2004; 30:293-8. [PubMed: 15173366]

Bhatti P, Kampa D, Alexander BH, Mcclure C, Ringer D, Doody MM, Sigurdson AJ. Blood spots as an alternative to whole blood collection and the effect of a small monetary incentive to increase participation in genetic association studies. BMC Med Res Methodol. 2009; 9:76. [PubMed: 19912630] 
Dlugos DJ, Scattergood TM, Ferraro TN, Berrettinni WH, Buono RJ. Recruitment rates and fear of phlebotomy in pediatric patients in a genetic study of epilepsy. Epilepsy Behav. 2005; 6:444-6. [PubMed: 15820358]

Doody MM, Sigurdson AS, Kampa D, Chimes K, Alexander BH, Ron E, Tarone RE, Linet MS. Randomized trial of financial incentives and delivery methods for improving response to a mailed questionnaire. Am J Epidemiol. 2003; 157:643-51. [PubMed: 12672684]

Engel J Jr. Mesial temporal lobe epilepsy: what have we learned? Neuroscientist. 2001; 7:340-52. [PubMed: 11488399]

Galea S, Tracy M. Participation rates in epidemiologic studies. Ann Epidemiol. 2007; 17:643-53. [PubMed: 17553702]

Groves RM, Cialdini RB, Couper MP. Understanding The Decision to Participate in a Survey. Public Opin Q. 1992; 56:475-495.

Haddow G. "We only did it because he asked us": gendered accounts of participation in a population genetic data collection. Soc Sci Med. 2009; 69:1010-7. [PubMed: 19666205]

Hara M, Higaki Y, Imaizumi T, Taguchi N, Nakamura K, Nanri H, Sakamoto T, Horita M, Shinchi K, Tanaka K. Factors influencing participation rate in a baseline survey of a genetic cohort in Japan. J Epidemiol. 2010; 20:40-5. [PubMed: 19897942]

Hauser WA, Annegers JF, Kurland LT. Incidence of epilepsy and unprovoked seizures in Rochester, Minnesota: 1935-1984. Epilepsia. 1993; 34:453-68. [PubMed: 8504780]

Hedera P, Blair MA, Andermann E, Andermann F, D'agostino D, Taylor KA, Chahine L, Pandolfo M, Bradford Y, Haines JL, Abou-Khalil B. Familial mesial temporal lobe epilepsy maps to chromosome 4q13.2-q21.3. Neurology. 2007; 68:2107-12. [PubMed: 17377072]

Hwang SK, Hirose S. Genetics of temporal lobe epilepsy. Brain Dev. 201110.1016/j.braindev. 2011.10.008

Johnson VA, Edwards KA, Sherman SL, Stephens LD, Williams W, Adair A, Deer-Smith MH. Decisions to participate in fragile $\mathrm{X}$ and other genomics-related research: Native American and African American voices. J Cult Divers. 2009; 16:127-35. [PubMed: 19824292]

Kaufman D, Murphy J, Scott J, Hudson K. Subjects matter: a survey of public opinions about a large genetic cohort study. Genet Med. 2008; 10:831-9. [PubMed: 19011407]

Kirkland SA, Raina PS, Wolfson C, Strople G, Kits O, Dukeshire S, Angus CL, Szala-Meneok K, Uniat J, Keshavarz H, Furlini L, Pelletier A. Exploring the acceptability and feasibility of conducting a large longitudinal population-based study in Canada. Can J Aging. 2009; 28:231-42. [PubMed: 19860978]

Murphy E, Thompson A. An exploration of attitudes among black Americans towards psychiatric genetic research. Psychiatry. 2009; 72:177-94. [PubMed: 19614555]

Murphy EJ, Wickramaratne P, Weissman MM. Racial and ethnic differences in willingness to participate in psychiatric genetic research. Psychiatr Genet. 2009; 19:186-94. [PubMed: 19593860]

Ottman R. Genetics of the partial epilepsies: a review. Epilepsia. 1989; 30:107-11. [PubMed: 2492220]

Ottman R. Progress in the genetics of the partial epilepsies. Epilepsia. 2001; 42(Suppl 5):24-30. [PubMed: 11887964]

Ottman R, Berenson K, Barker-Cummings C. Recruitment of families for genetic studies of epilepsy. Epilepsia. 2005; 46:290-7. [PubMed: 15679510]

Ottman R, Hauser WA, Stallone L. Semistructured interview for seizure classification: agreement with physicians' diagnoses. Epilepsia. 1990; 31:110-5. [PubMed: 2406127]

Ottman R, Lee JH, Hauser WA, Hong S, Hesdorffer D, Schupf N, Pedley TA, Scheuer ML. Reliability of seizure classification using a semistructured interview. Neurology. 1993; 43:2526-30. [PubMed: 8255451]

Ottman R, Lee JH, Risch N, Hauser WA, Susser M. Clinical indicators of genetic susceptibility to epilepsy. Epilepsia. 1996; 37:353-61. [PubMed: 8603640]

Remington TD. Telemarketing and Declining Survey Response Rates. J Advert Res. 1992; 32:Rc6Rc7. 
Rylander-Rudqvist T, Hakansson N, Tybring G, Wolk A. Quality and quantity of saliva DNA obtained from the self-administrated oragene method--a pilot study on the cohort of Swedish men. Cancer Epidemiol Biomarkers Prev. 2006; 15:1742-5. [PubMed: 16985039]

Schleifer S. Trends in Attitudes Toward and Participation in Survey Research. Public Opin Q. 1986; 50:17-26.

Vadlamudi L, Scheffer IE, Berkovic SF. Genetics of temporal lobe epilepsy. J Neurol Neurosurg Psychiatry. 2003; 74:1359-61. [PubMed: 14570824]

Winawer MR, Rabinowitz D, Barker-Cummings C, Scheuer ML, Pedley TA, Hauser WA, Ottman R. Evidence for distinct genetic influences on generalized and localization-related epilepsy. Epilepsia. 2003; 44:1176-82. [PubMed: 12919389]

Winawer MR. Phenotype definition in epilepsy. Epilepsy Behav. 2006; 8:462-76. [PubMed: 16497563] 
REASONS FOR INELIGIBILITY

$\square$ ACQUIRED/SYMPTOMATIC EPILEPSY $\quad$ SINGLE UNPROVOKED SEIZURE

AGE OF ONSET OVER $35 \quad$ NOT SEIZURES

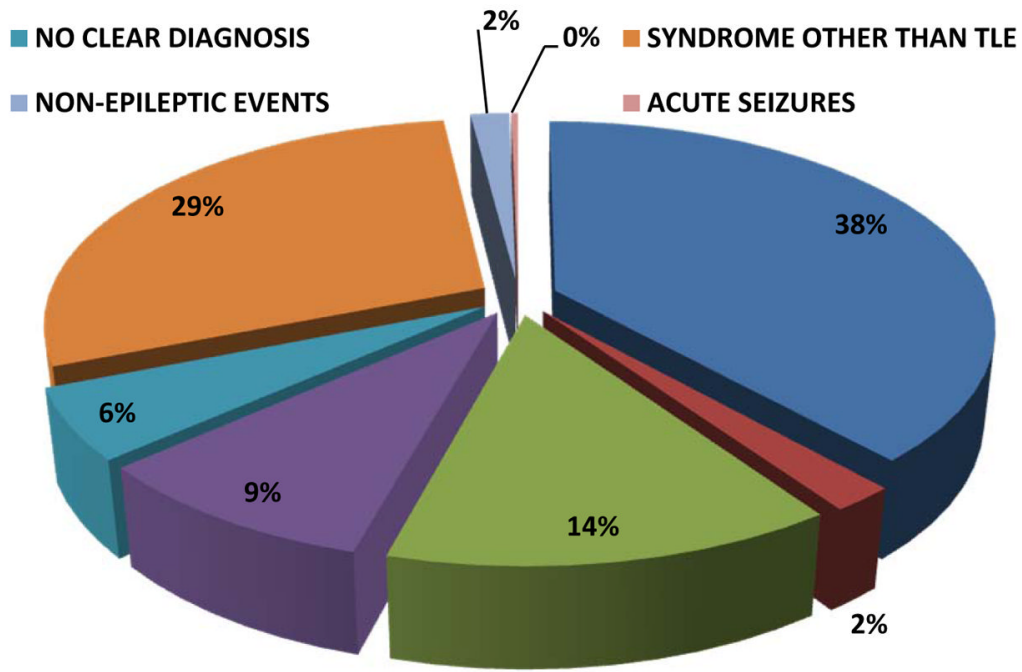

Figure 1.

Reasons for exclusion 


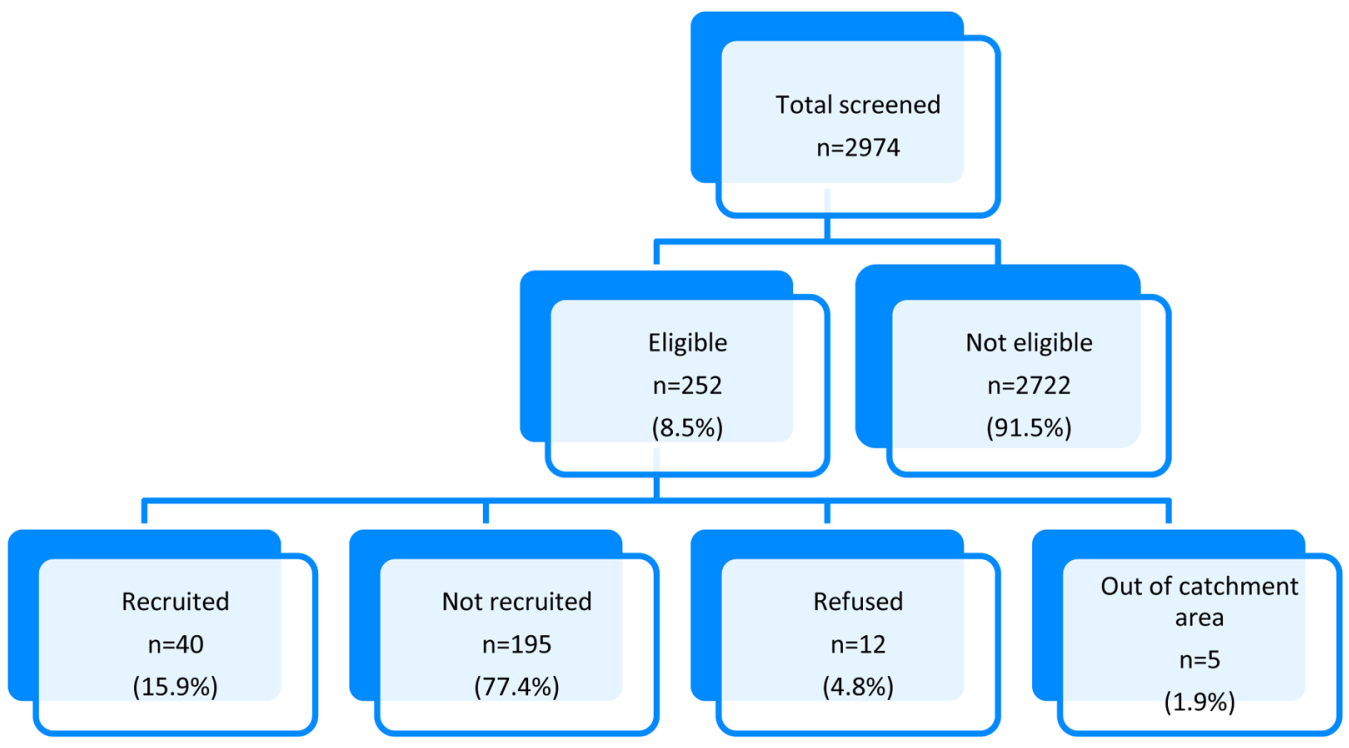

Figure 2.

Outcome of total participants screened 
